\title{
Fetal Heart Rate Decelerations Evaluated by Hypoxia Index
}

\author{
Kazuo Maeda* \\ Department of Obstetrics and Gynecology, Tottori University Medical School, Japan
}

*Corresponding author: Kazuo Maeda, Department of Obstetrics and Gynecology, Emeritus, Tottori University Medical School, Tottoriken, Japan

Submission: 卌 October 12, 2017; Published: 监 March 12, 2018

\begin{abstract}
As numeric objective decision was needed in computerized FHR analysis, FHR score, hypoxia index, actocardiographic A/B ratio and frequency spectrum analysis were introduced into computerized FHR diagnosis, which brought improved perinatal outcome. Final computer output is objective but clinical, namely, not only FHR parameters but also clinical Apgar score is directly and rapidly reported to the attendant doctor, who is possible to manage the fetus with these objective data.
\end{abstract}

Keywords: Fetal monitoring; Computer; FHR Score; Hypoxia index; Frequency spectrum; A/B Ratio of actocardiagram

\section{Introduction}

Fetal heart rate patterns, including early, late and variable decelerations $[1,2]$ could be detected by computer, however, fetal outcome was expected by doctor's subjective decision, including controversy interpretation and observer differences in FHR pattern analysis, therefore, numeric and objective method was mandatory by computer analysis of FHR changes, where clinical Apgar score and UApH were predicted using regression equations of computer diagnosis, Apgar score and UApH obtained in FHR and neonatal studies, then numeric outcome is obtained automatically, where long-term outcome numerization was needed to diagnose the outcome [3]. Attending doctor should receive not only mechanical results of computer output but also clinical Apgar score obtained using regression equation, e.g. FHR score as well as predicted Apgar score, umbilical arterial blood pH(UAPH) and outcomes directly and rapidly by cellular phone or computer local area network (LAN), which promoted correct fetal management.

\section{Methods}

Primarily an obstetrician should prepare an obstetrically specialized computer [4], which is connected to a fetal heart rate (FHR) monitor, which will be autocorrelation FHR monitor triggered by ultrasonic Doppler fetal cardiac signal or heart tone, but it is better to prepare an actocardiograph, which records FHR, fetal movements and uterine contraction [5].

\section{Objective numeric analysis of fetal heart rate}

The fetal monitor output signals are digitized by analog-digital converter, and its details are analyzed preparing further analysis including FHR score, hypoxia index, A/B ratio, etc.

\section{FHR score}

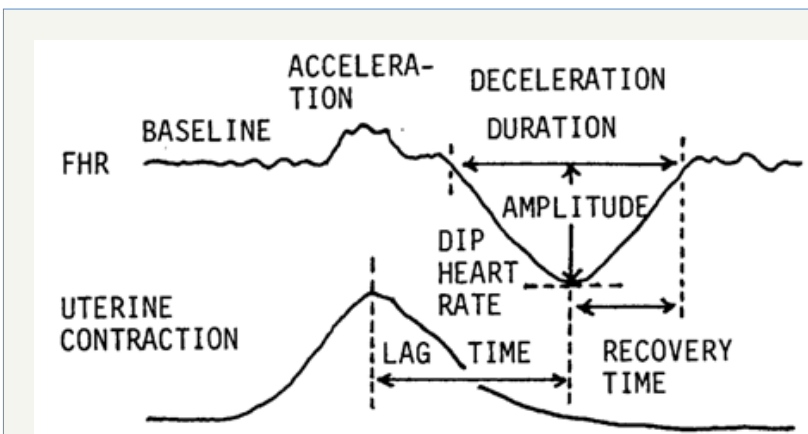

Figure 1: Numeric measurements of fetal heart rate record in the calculation of FHR score estimating the fetal outcome.

Table 1: Evaluation scores to calculate FHR score.

\begin{tabular}{|c|c|}
\hline FHR changes & Evaluation \\
\hline Baseline $110-130$ or $160-180 \mathrm{bpm}$ & 1 \\
\hline$<110$ or $>180 \mathrm{bpm}$ & 3 \\
\hline Deceleration The lowest FHR $<100 \mathrm{bpm}$ & 2 \\
\hline Amplitude $>50 \mathrm{bpm}$ & 2 \\
\hline Duration $>60 \mathrm{sec}$ & 2 \\
\hline Lag time $>40 \mathrm{sec}$ & 3 \\
\hline Recovery time $>40 \mathrm{sec}$ & 3 \\
\hline Accompany no acceleration & 2 \\
\hline W shape & 4 \\
\hline
\end{tabular}


The sum of evaluations in $5 \mathrm{~min}$ is FHR score [2].

1st stage FHR score <10: normal neonate

=10: predicted Apgar $=6$

$=15$; predicted Apgar $=4$ and acidosis

FHR changes are evaluated in 5 min analyzing FHR baseline and decelerations, where detailed data of FHR baseline and deceleration are obtained (Figure 1). The evaluation scores determined according to the percentages of Apgar scores, which was lower than 7, in each parameters (Table 1), the sum of evaluation scores in $5 \mathrm{~min}$ is the FHR score, which was calculated by the computer.

Regression equations were obtained between FHR score and neonatal Apgar score and UAPH. The results of new FHR score and regression equations are reported automatically by the computer (Table 2) [4]. Expected Apgar score and UApH are studied by attending doctor and any treatment including early fetal delivery with caesarean delivery (CD) is indicated. Regression equations are as follows:

Table 2: Apgar score and UApH are predicted by following regression equations and reported to the doctor.

FHR score =10; Newborn is expected to be mild asphyxia, normal pH

FHR score $=15$, Newborn is expected to be asphyxia, $\mathrm{pH}$ is acidosis

FHR score $=20$, Newborn is expected severe asphyxia, $\mathrm{pH}$ is acidosis

Apgar score $=9.361-0.335 \times F H R$ score

$\mathrm{UApH}=7.31-0.016 \times$ FHR score.

Predicted Apgar score $=9.361-0.335$ x FHR score

Predicted UApH=7.31-0.016 x FHR score

\section{Hypoxia index}

The late deceleration (LD) was reported to be ominous in FHR pattern classification, however, fetal outcome was favorable, if $3 \mathrm{LDs}$ preceded CD, and fetal outcome was ominous, including the loss of variability, severe neonatal asphyxia, low Apgar score, UApH acidosis, infantile brain damage and cerebral palsy, when the LD highly repeated. In addition, some researchers did not defined before its repetition for $15 \mathrm{~min}$, namely, repetition of deceleration is mandatory to develop hypoxic effect on the fetus. The repetition is measured by the length of the duration of low $\mathrm{PaO}_{2}$, Thus, the author intended to measure the sum of duration of length of low $\mathrm{PaO}_{2}$. However, it is uncommon to sample fetal blood in the labor.

I remembered the correlation of heart rate to $\mathrm{PaO}_{2}$ in rabbit [6], limitedly in the area where $\mathrm{PaO}_{2}$ was $50 \mathrm{mmHg}$ or less. Fortunately, human umbilical arterial $\mathrm{PaO}_{2}$ was $50 \mathrm{mmHg}$ or less [7]. Thus, the author measured the duration of FHR deceleration instead of low $\mathrm{PaO}_{2}$ duration, where the intensity of hypoxia was represented by the lowest FHR, then hypoxia index (HI) was formed as follows

Hypoxia index $(\mathrm{HI})=$ The sum of deceleration duration (min) divided by the nadir FHR, and multiplied by 100 .

The author had two cases of fetal brain damage followed by cerebral palsy in one and another was 50min repetition of LD (Figure 2), whose Apgar was 3 and the baby died by the brain hemorrhage 3 months after birth. Both associated the loss of variability similar to an anencephalic fetus. The author measured their HIs, where the first one was 25 and another 26, while HI was 20 to 24 in cases who revealed abnormal FHR but maintained baseline variability without any brain damage [8]. Thus, the threshold HI was 25 to develop cerebral palsy. Therefore, it was recommended to deliver the fetus before the loss of variability [9], when the HI is less than 25 . The other sign of early delivery to prevent cerebral palsy is the loss of acceleration and decreased variability to $5 \mathrm{bpm}$, Regression equation of hypoxia index in 6 cases;

Expected Apgar score $=10.34-0.28 \mathrm{HI}$

$$
\mathrm{R}^{2}=0.97, \mathrm{P}<0.01
$$

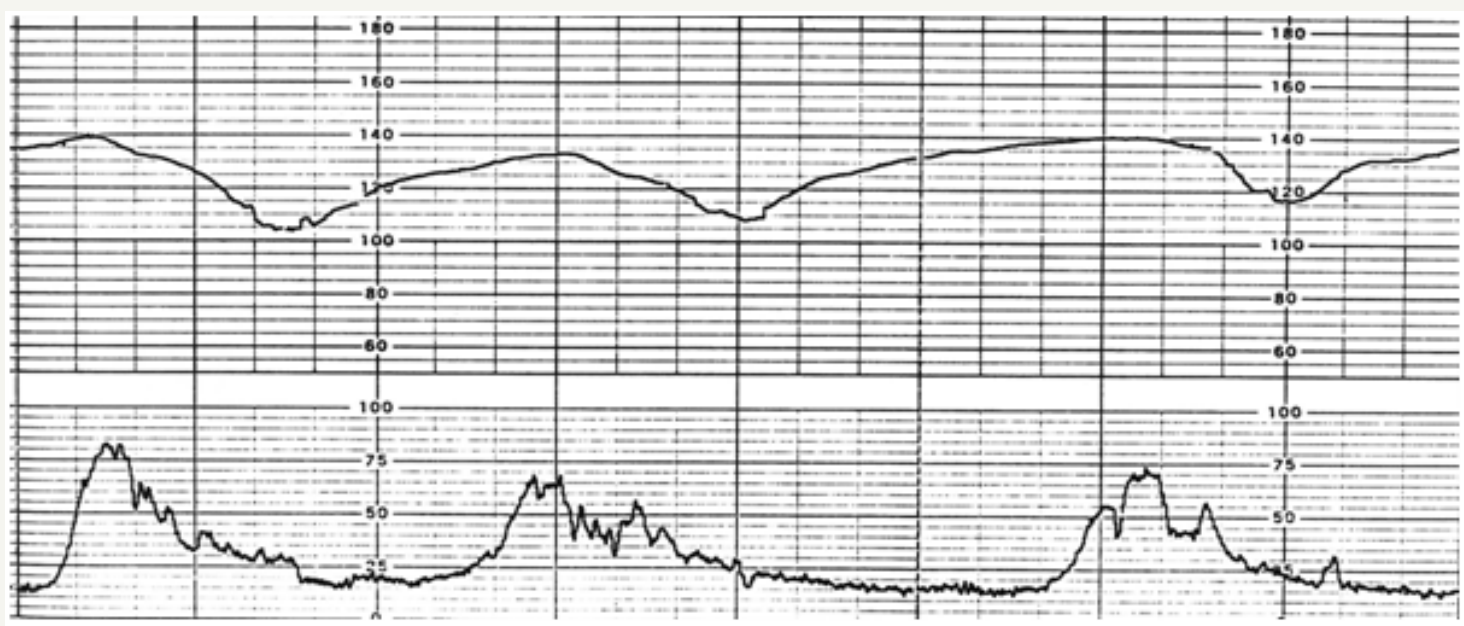

Figure 2: Typical late decelerations the lag time between the peak of uterine contraction and the nadir of deceleration was 60 sec. The LDs were repeated for $50 \mathrm{~min}$, due to refusal of caesarean delivery. The hypoxia index was 26 and the LD was associated the loss of FHR variability, and heavy brain damage. 


\section{Actocardiographic A/B ratio}

It is the sum of FHR acceleration durations, divided by the sum of fetal movement burst durations in a definite period of FHR monitoring with actocardiogram.

Apgar score is 7 or more, when A/B ratio is 1 or more.

Apgar score is $<7$, when A/B ratio is less than 1 [10].

A/B ratio and predicted Apgar score are directly reported to attendant doctor.

\section{FHR chart record}

As no FHR pattern was studied in fetal diagnosis in numeric computerized FHR, there was no chart recorder in the centralized computer system, but the computer hard copy traced FHR curve when it is ordered in necessary case. It was particularly useful computer function to directly and quickly report risky and hazardous changes to attending doctor before or at the fetal hypoxic damage using objective data (Table 3) [11].

Table 3: Regession equation of hypoxia Index and Apgar score.

\begin{tabular}{|c|}
\hline HI=6, Newborn is normal \\
\hline HI=12, Newborn is moderate asphyxia. \\
\hline $\mathrm{HI}>=25$, Newborn is severe asphyxia, and the brain is damaged \\
\hline
\end{tabular}

Where Apgar score $=10.34-0.28 \times$ hypoxia index .

\section{Results}

Apgar score and umbilical arterial $\mathrm{pH}(\mathrm{UApH})$ are predicted by FHR score in the $1^{\text {st }}$ stage of labor by their regression equations;

Predicted Apgar score $>=7$, if FHR score $<10$

$=6$, if FHR score is 10,

$=4$, if FHR score is 15

The neonate was acidosis, if FHR score $>=15$.

Thus, Caesarean delivery is recommended if FHR score is 15 or more in the 1st stage of labor.

Caesarean delivery was also recommended when hypoxia index is 20-23, where FHR acceleration is lost and the variability reduced to $5 \mathrm{bpm}$, which were the states immediately before the loss of variability followed by cerebral palsy [7].

\section{Improved perinatal states in the update computerized fetal monitoring}

Three systems were compared by Dr. Utsu, in no computer period, centralized computer without direct reporting to doctor, and update system in 2012, in which directly and quickly reported FHR abnormality to the doctor. There was significant decrease of perinatal mortality and no case of cerebral palsy in the update system, i.e. the advantage was recognized in the update system [11].

\section{Frequency spectrum analysis}

Pathologic sinusoidal FHR, which was severe fetal anemia close to fetal death, was diagnosed by the FHR frequency spectrum analysis of FHR, when the La/Ta ratio was $30 \%$ or more and PPSD was $300 \mathrm{bpm}^{2} / \mathrm{Hz}$ or more [8]. In addition, the loss of baseline variability was diagnosed, when La/Ta ratio was less than $15 \%$ and the PPSD was less than $60 \mathrm{bpm}^{2} / \mathrm{Hz}$ [9]. Clinical diagnosis is reported doctor.

\section{Special character of fetal hiccupping movements}

Fetal hiccup pings are recorded as sharp spikes in actocardiagram, of which intervals were regularly 2-3 sec. It repeats for more than $10 \mathrm{~min}$ without movement burst. It does not associate acceleration, while no hypoxia was found, because it will be local diaphragmatic contraction. No FHR acceleration is detected against sharp fetal movement spiks with 2 sec interval, and no FHR change was detected by computer analysis as neither deceleration nor acceleration with moderate variability developed, no pathologic change was diagnosed.

\section{New clinical fetal monitoring computer}

FHR score software had been completed and it is working in fetal monitoring. In hypoxia index, duration of deceleration and nadir heart rate are found in the computerized calculation of FHR score [11], which was limited in 5 min, while hypoxia index is determined in full course of fetal monitoring. A/B ratio was already studied and reported [10]. Frequency spectrum software was completed and utilized in central computerized FHR analysis, and direct reporting system was successful in multiple delivery monitoring [11]. All components of this report were completed and we are planning a new personal system. Maternal posture in late deceleration the mother of fetal late deceleration (LD) should try lateral posture, where LD disappeared when it was caused by the iliac arterial compression by contracted uterus reducing placental maternal blood flow [12].

\section{Conclusion}

FHR score, hypoxia index, A/B ratio and frequency spectrum are main functions of computerized numeric FHR analysis. FHR pattern classification was absorbed into hypoxia index analysis. A computer connected to actocardiogram is the simple setting of numeric analysis, and multiple time sharing system, handling 50 deliveries at the same time, is used in large hospital. Medical diagnosis is reported doctor instead of computer terminology.

\section{References}

1. Hon EH (1968) An atlas of fetal heart rate patterns. Harty Press, New Haven, USA.

2. Barcia RC, Poseiro JJ, Bauer CM, Gulin LO (1967) Effects of abnormal uterine contraction on fetal heart rate during labor. Proc $5^{\text {th }}$ World Cong Gynecol Obst, Sydney.

3. Maeda K, Nagasawa T (2010) Loss of FHR variability diagnosed by frequency analysis. J Perinat Med 38(2): s197-201.

4. Maeda K, Noguchi Y, Nagasawa T (2006) Quantitative fetal heart rate evaluation without pattern classification. In: Kurjak \& Chervenak (Eds.), Textbook of Perinatal Medicine, ( $2^{\text {nd }}$ edn), Volume 2, Informa, UK, pp. 1487-1495. 
5. Umezawa J (1976) Studies on the relation between heart rate and $\mathrm{PaO}_{2}$ in hypoxic rabbit a comparative study for fetal heart rate change during labor. Acta Obstet Gynecl Jpn 28: 1203-1212.

6. Maeda K (2016) Actocardiogram, analysis of fetal motion and heart rate. The Health Sciences Publisher, London, UK.

7. Maeda K, Kimura S, Nakano H (1969) Pathophysiology of Fetus. Fukuoka Printing, Fukuoka, Japan.

8. Maeda K (2014) Modalities of fetal evaluation to detect fetal compromise prior to the development of significant neurological damage. J Obstet Gynaecol Res 40(10): 2089-2094.

9. Maeda K, Nagasawa T (2005) Automatic omputerized diagnosis of fetal sinusoidal heart rate. Fetal Duag Ther 20(5): 328-334.

10. Maeda K, Iwabe T, Ito T, Pooh RK, Minagawa Y, et al. (2009) Detailed multigrade evaluation of fetal disorders with the quantified actocardiogram. J Perinat Med 37(4): 392-396.

11. Maeda K, Noguchi Y, Nagasawa T, et al. (2012) Central computerized automatic fetal heart rate diagnosis with a rapid and direct alarm system. The Open Medical Devices Journal 4: 28-33.

12. Poseiro JJ, Bauer CM, Barcia RC (1969) Effect of uterine contractuions on maternal blood flow through the placenta. Perinatal factors affecting human development. Pahoa Advisory Committee 161-171.
Creative Commons Attribution 4.0

International License

For possible submissions Click Here

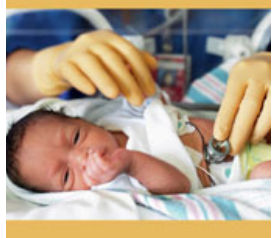

Research in Pediatrics \& Neonatology

\section{Benefits of Publishing with us}

- High-level peer review and editorial services

- Freely accessible online immediately upon publication

- Authors retain the copyright to their work

- Licensing it under a Creative Commons license

- Visibility through different online platforms 